\title{
The Free Form Deformation of Phytoplankton Models
}

\author{
Anton Lyakh \\ Dept. of Biophysical Ecology, Institute of Biology of the Southern Seas, 2, Nakhimov av., \\ Sevastopol, 99011, Ukraine \\ anton@ibss.iuf.net, antonberlin@yahoo.com
}

\begin{abstract}
We construct the 3D models of phytoplankton. In presented article we solve the problem about deformation of the created models: How to deform the model so that its shape should conform to the shape of the natural microorganism? We use the Free Form Deformation (FFD) for the solving of this problem. In the first case, when the dimensions of the microorganism are given, the distances between control points of the FFD are equated to these dimensions. In the second case, when a scientist has the image of the microorganism, he should deform the model, displacing the control points, so that the image edge and the model's outer border has coincided. The control points location is used in the studying morphological changes of microorganisms and describing their shapes.
\end{abstract}

\section{Introduction}

The oceanic microorganisms (bacteria, phytoplankton) are the important part of the World Ocean food web, without which the life in the ocean will die out. Microorganisms' tiny dimensions (less than $1 \mathrm{~mm}$ ) produce difficulties for their studying.

The volume and surface area of microorganisms need to be measured practically at all microbiological researches. Not only the tiny dimensions of microorganisms complicate the solution of the given problem, but also by the complex shape of their body. Till now the biologists approximate the shape of phytoplankton by a set of simple geometrical primitives, such as a sphere, a cone, an ellipsoid, etc. (Fig. 1). It is clearly that at such approach the results of calculation of the volume and the surface area of phytoplankton cell (microalgae) will be insufficiently exact. At the same time application of 3D modeling will allow to precisely approximate the shape of the cells and to receive more authentic evaluations of their volume and surface area. Besides this the $3 \mathrm{D}$ model of the microorganism will allow studying its morphological features in detail.

The main stages of the construction of the 3D phytoplankton cell are first described by Lyakh, et. al. in 2001 [1]. The main problem in 3D cell building is the fitting of the model shape to the shape of natural microalgae. For the solution of the given problem we determine the location of control points, which are the control points of Free Form Deformation (FFD), on the model surface. The displacement of the control points 

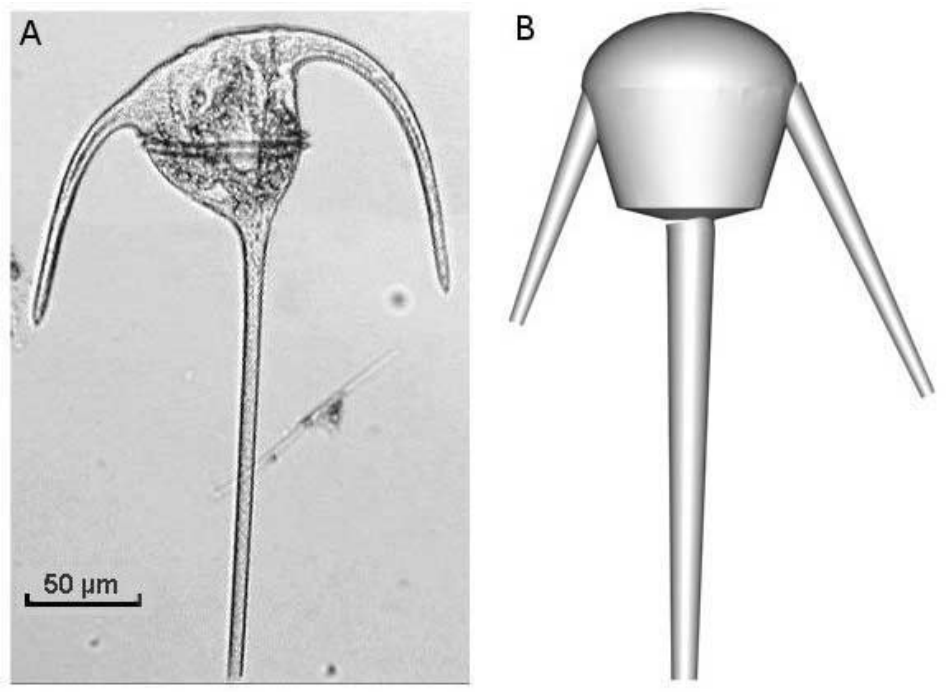

Fig. 1. The comparison of the phytoplankton cell's body shape with the geometric model, which is used for the cell volume and surface area calculation. A - the microphotograph of the phytoplankton cell Ceratium tripods; B - the 3D model created from different geometric primitives. In many cases the 3D model can be simpler

produces the deformation of the model that allows fitting the model shape to the shape of the natural cell.

\section{Free Form Deformation}

FFD is defined by parametric functions (3D splines) whose values are determined by the location of the control points (CP) of deformation lattice (Fig. 2). Once the control points are moved, the new location of object vertexes is determined by the weighted sum of the control points [2].

The FFD is a mapping operating from the world space of the model to the local space of the deformation lattice, and conversely to the world space:

$$
\text { FFD: } \mathrm{R}^{3} \rightarrow \mathrm{R}^{3} \rightarrow \mathrm{R}^{3}
$$

Let function $\mathrm{F}: \mathrm{R}^{3} \rightarrow \mathrm{R}^{3}$ maps the vertex $\mathrm{X}=(x, y, z)$ of the object in the world space to the vertex $\mathrm{U}=(u, v, w)$ of the object in the space of deformation lattice:

$$
\mathrm{F}(\mathrm{X})=\mathrm{U} \text {. }
$$

Let function $\mathrm{F}^{\prime}: \mathrm{R}^{3} \rightarrow \mathrm{R}^{3}$ maps the vertex $\mathrm{U}=(u, v, w)$ to the vertex $\mathrm{X}^{\prime}=\left(x^{\prime}, y^{\prime}, z^{\prime}\right)$ of an object in the deformed world space:

$$
\mathrm{F}^{\prime}(\mathrm{U})=\mathrm{X}^{\prime} \text {. }
$$




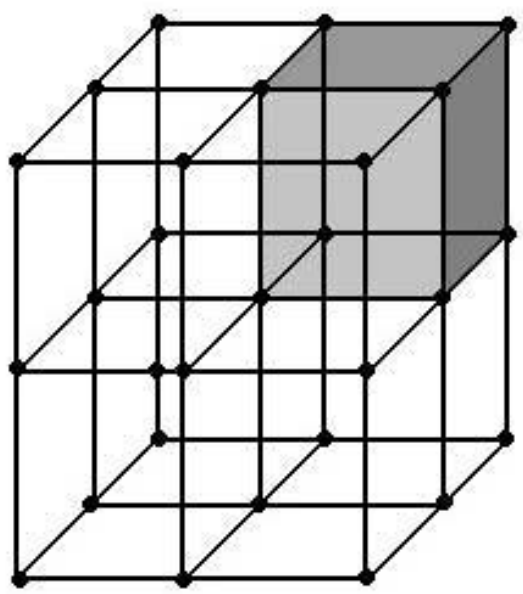

Fig. 2. The deformation lattice: black circles are the control points (or the knots) of the deformation lattice, gray box is the deformation cell

Then the composition of these functions defines FFD:

$$
\operatorname{FFD}(X)=F^{\prime}(F(X))=X^{\prime} \text {. }
$$

The form of the deformation lattice defines the function $\mathrm{F}$, which assigns to each vertices of the object a set of local coordinates. We use the local parallelepiped-shape coordinate system of the deformation lattice. Therefore $\mathrm{F}$ is the transformation of the world coordinate system to the coordinate system of a parallelepiped, which sides are considered equal to unit length.

The function $\mathrm{F}^{\prime}$ is a sum of control points $\mathrm{P}_{\mathrm{i}, \mathrm{j}, \mathrm{k}}$ weighted by polynomial basis functions $\mathrm{B}_{\mathrm{i}}(\cdot)$ :

$$
F^{\prime}(u, v, w)=\sum_{i=0}^{N i} \sum_{j=0}^{N j} \sum_{k=0}^{N k} B_{i, N i}(u) \cdot B_{j, N j}(v) \cdot B_{k, N k}(w) \cdot P_{i . j . k} \cdot
$$

The basis functions B are defined as follows:

$$
\mathrm{B}_{\mathrm{i}, \mathrm{N}}(\mathrm{u})=\mathrm{C}_{\mathrm{N}}^{\mathrm{i}} \cdot(1-\mathrm{u})^{\mathrm{N}-\mathrm{i}} \cdot \mathrm{u}^{\mathrm{i}}
$$

FFD proceeds in three steps:

1. Object vertices are assigned local coordinates $u, v, w$ ( $\mathrm{F}$ is applied). Since the local parallelepiped has sides with unit length, therefore the local coordinates $u, v, w$ varies from 0 to 1 .

2. Control points are displaced that cause the distortion of the local space. But the model's local coordinates don't change. They never change.

3. Equation 5 is applied to all of the object vertices to produce the deformation of the model (Fig. 3). 

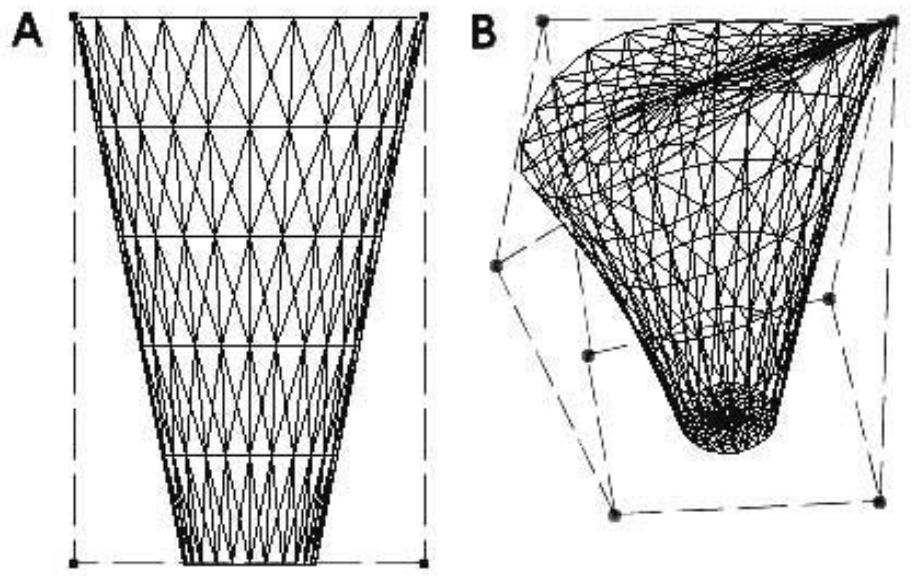

Fig. 3. The FFD of the cone: black circles are the FFD control points; dashed lines show the boundaries of parallelepiped-shape coordinate system. A - the source object; B - displacement of the control points causes the deformation of the cone

\section{The Deformation of the Phytoplankton Model}

The location of the control points is the important factor influenced on the model's deformation. Each control point influences only on adjacent deformation cells. Therefore, the dimension of deformation cells is the important factor too. The dimension of deformation cells determines a region, which will be deformed. Finally, the application of the one FFD operator to whole model will essentially hamper the process of model deformation. Therefore, the cell's model is divided into parts (elements), and a single FFD operator is applied to each model's part.

\subsection{The location and classification of the control points}

The initial location and amount of $\mathrm{CP}$ plays the defining role during the model deformation. With $\mathrm{CP}$ number increase the ability of the model to accept the necessary shape is increased. However, many $\mathrm{CP}$ will hamper the deformation.

The location of the control points. Not all control points of the model have an analog on the natural object. The location of the majority of CP is chosen so that the model of the microalgae would be convenient for deforming. Therefore control points are located on the following parts of the cell:

- One control point is located at concave and convex parts of the cell, at the top of the cell's growth, etc. (Fig. 4, A). 

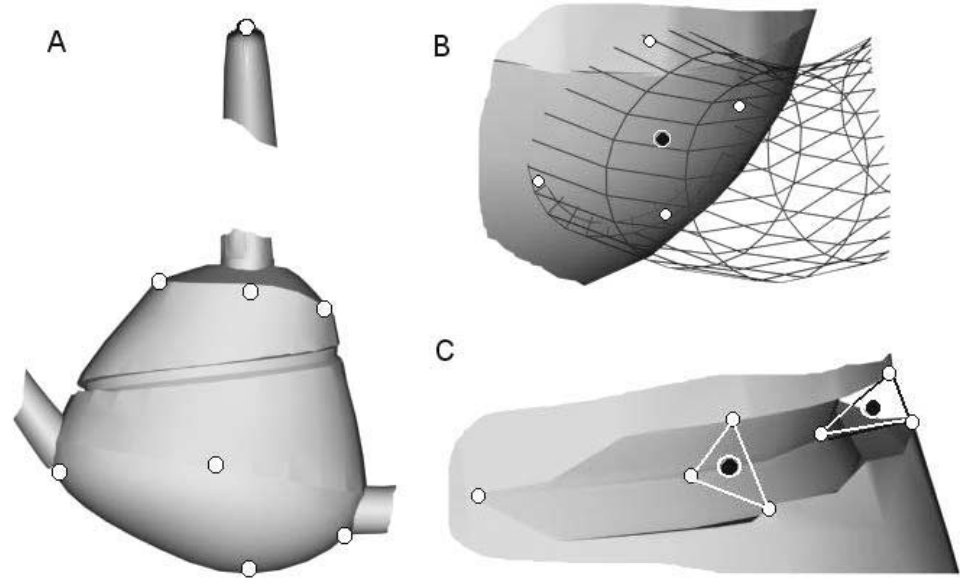

Fig. 4. The location of the control points $(\mathrm{CP})$ on the different parts of phytoplankton cell. A on the cell's growth and on the cell's body; B - in the zone of contact the cell's element with the cell's body; C - on the cell's groove: the groups of points, which are put along the groove's edges, are delineated by triangles; white circles are the ordinary $\mathrm{CP}$; black circles are the main $\mathrm{CP}$ (see the text for detail)

- Five CP are located at the places where the cell's elements contact with the cell's body. One $\mathrm{CP}$ is located at the center of the contact zone; another four are located on the edges of the contact zone (Fig. 4, B).

- The group of the control points is uniformly distributed along the edges of the cell's grooves and keels. Two points are located at the groove/keel ends; two points at the groove/keel edges; one point in the hollow/crest; and the last one in the center of the formed triangle (Fig. 4, C).

The classification of the control points. The deformation of the cell element consists of the distortion of the element's surface and the deformation of element's medial axis. The distortion of the element's surface is caused by the displacement of the ordinary control points, which are situated on it (white circles on Fig. 4). The changing of the ordinary $C P$ position produces the immediate deformation of the model. The deformation of the element's medial axis is caused by the displacement of the main control points, which are situated in the center of the contact zone, or along the axial line of the cell's groove/keel (black circles on Fig. 4). The main control point is connected with the ordinary control points. The changing of the main CP position produces the shift of the ordinary $C P$ that causes the deformation of a model.

That is, the difference between the ordinary and the main $\mathrm{CP}$ is that the first one makes the direct deformation of the model, and the second one makes the indirect model deformation, by means of the first. Using the two types of the control points facilitates a 3D model deformation. 

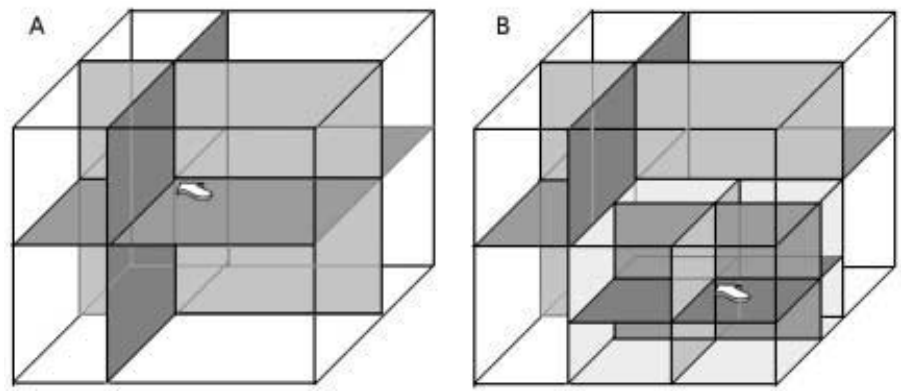

Fig. 5. The demonstration of the algorithm of the deformation lattice construction. White arrows show the active control point. The points of the intersection of the lines are the knots, which cannot be displaced by a scientist. A - the first step: three planes are passed through the $\mathrm{CP}$ till they reach the bounding parallelepiped; $\mathrm{B}$ - the following steps: three planes are passed through the $\mathrm{CP}$ till they reach another planes

\subsection{The construction of the deformation lattice}

It is important to determine dimensions of deformation cell so that the displacement of the control points not cause the effect unnecessary to a user. If the user expects the deformation of certain model's part, that this model's part must be deformed.

The traditional method of the deformation lattice construction not provides performance of this task. The traditional method assumes that the deformation cells have equal dimensions, and they are uniformly distributed inside the parallelepiped, which bounds the model (Fig. 2). Similar construction of the deformation lattice has the fault. In most cases the position of the deformation lattice knots not always coincides with the position of the model's control points. Therefore, for the movement of these control points it is necessary to displace the knots of the deformation lattice. But we must displace the control points not the knots. Therefore the position of the knots must coincide with the position of the control points. We use the following method of the deformation lattice construction, which provides the coincidence of the knots with the control points:

1. The bounded parallelepiped is circumscribed around the model, and an arbitrary control point is selected.

2. Three planes, parallel to the parallelepiped sides, are drawn through the first control point up to the bounds of the parallelepiped (Fig. 5, A).

3. The next control point is selected, and three planes, parallel to the parallelepiped sides, are drawn through this control point too. The planes are drawn up to intersection with other planes (Fig. 5, B).

4. Step three is repeated for each control point.

During the deformation lattice construction the new knots are created (the points of the intersection of the lines on Fig. 5, which are not marked by arrows). But these knots are not the control points. A user can't displace they. These knots only determine the bounds of deformation cells. 

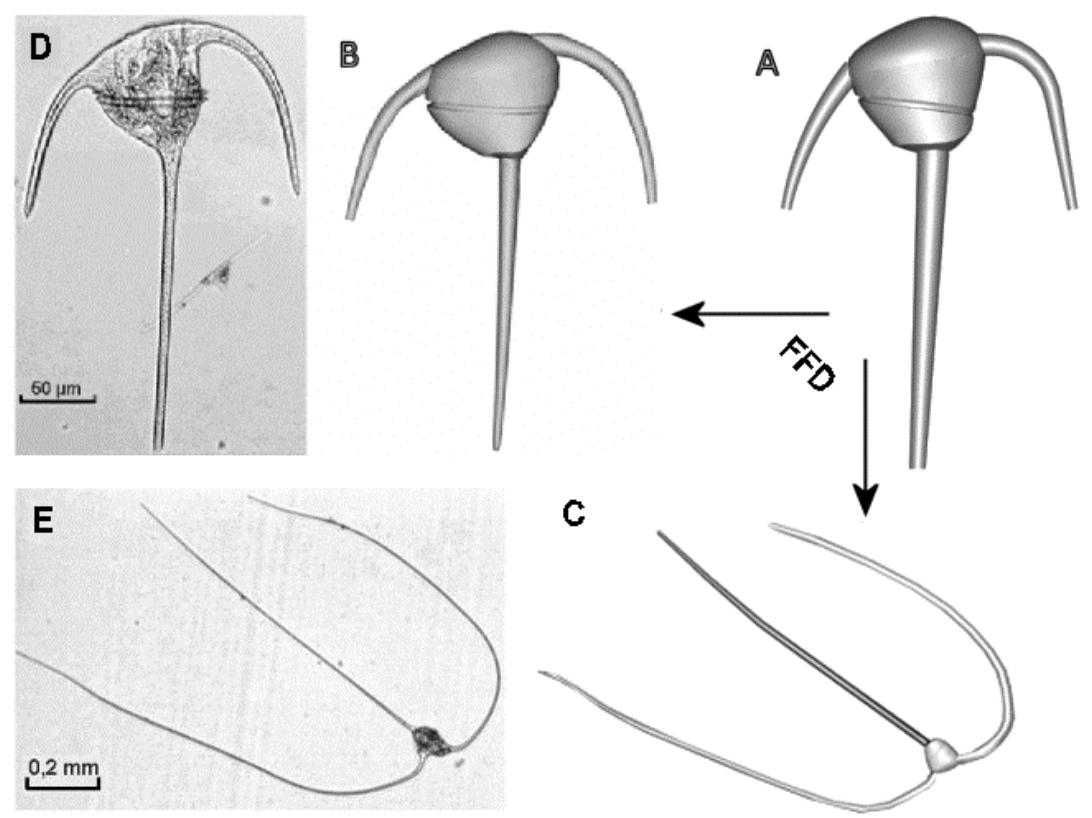

Fig. 6. Some examples of using the FFD operator to phytoplankton cells modeling. A - the base model, which is deformed by the user; B,C - the 3D models of phytoplankton cells created by the FFD procedure; D,E - the microphotographs of phytoplankton cell Ceratium tripods (D), and Ceratium sp. (E), which are used as patterns for the fitting of the base model

Besides the construction of the deformation lattice we apply several FFD operators to each element of a phytoplankton cell. The division of the cell's model into the single elements executes in accordance with the classification of microalgae parts used in biology. For example, the 3D model on Fig. 1 consists of four elements: the body, two lateral growths, and one apical growth. If one FFD operator is applied to whole model, the process of model deformation will be essentially hampered. On the other hand, if the single FFD operator is applied to the each model parts, the process of the model deformation will become easier.

\subsection{Two methods of the phytoplankton model deformation}

The FFD procedure is used in the construction of the phytoplankton models of those cells, which are observed through a microscope. The images of phytoplankton cells are used as patterns of their shapes. The process of the model deformation consists of the sequential movements of the control points (an application of the FFD operator).

The FFD is applied to the model till a correspondence between the model shape and the natural microorganism shape is achieved.

But how a scientist can achieves this correspondence? The initial data determines the methods of this problem solving: 
1. In the first case, when the user cannot obtain the image of the phytoplankton cell, but can measure its dimensions, the measured sizes are used as the values of distances between the control points of the model. The setting of the distances causes the deformation of the model.

2. In the second case, when the user obtains the image of the phytoplankton cell, he should achieve coincidence of the model's borders with the outline of the microorganism on the image. The coincidence is achieved by displacing model's control points.

These two methods are the main methods of the application of the FFD operator to the phytoplankton cells modeling (Fig. 6). Initially a scientist has the base model of the phytoplankton cell, which represents an average shape of single phytoplankton species or genus. After that the user gets the initial data, deforms the base model, and obtains the model of specific phytoplankton species. Using this specific model he calculates the volume and the surface area of the phytoplankton cell and makes its morphological analysis. Undoubtedly, calculated surface and volume values will most exact.

\section{Conclusion}

The considered procedure of the phytoplankton models deformation is the main stage of the 3D phytoplankton cell building. The application of 3D simulation in biology allows receiving exact evaluations of the microorganisms' volumes and surface areas. The application of the FFD to the 3D phytoplankton model allows demonstrating microorganism morphological changes during its life cycle. The location of the phytoplankton model control points describes the shape of the cell, and it may be used at cell morphological analysis.

\section{Acknowledgments}

I express my thanks to Vladimir Mukhanov who has helped me during thinking over this paper.

\section{References}

1. Lyakh, A.M., Mukhanov, V.S., Kemp, R.B.: The Virtual Cell Project for the Investigation of Microalgal Morphology and Dispersity of Natural Phytoplankton. In: $17^{\text {th }}$ European Workshop on Computational Geometry (CG 2001). Program \& Abstracts (Freie Universität Berlin, March 2001). Berlin (2001) 57-58

2. Sederberg, T.W., Parry, S.R.: Free-Form Deformation of Solid Geometric Models. Computer Graphics 20 (1986) 151-160 